\title{
Um acervo centenário: a história da Coleção de Formigas (Insecta: Hymenoptera: Formicidae) do Museu de Zoologia da Universidade de São Paulo A centenary collection: the Ant Collection of the Museu de Zoologia da Universidade de São Paulo
}

\author{
Mônica Antunes Ulysséa' (1) Carlos Roberto Ferreira Brandão' \\ IMuseu de Zoologia da Universidade de São Paulo. Laboratório de Sistemática, \\ Evolução e Biologia de Hymenoptera. São Paulo, São Paulo, Brasil
}

\begin{abstract}
Resumo: A Coleção de Formigas do Museu de Zoologia da Universidade de São Paulo (MZSP) representa uma amostra inestimável da biodiversidade de formigas, principalmente da região neotropical. Seu acervo foi formado a partir da contribuição de naturalistas-viajantes, de mirmecólogos reconhecidos por suas contribuições científicas, do desenvolvimento de pesquisas e por sua atuação como instituição fiel depositária. Abriga uma expressiva quantidade de material-tipo, comporta 4.741 espécimes-tipos de 892 espécies, amplamente distribuídos. A prática curatorial constante e adequada é um dos grandes desafios da coleção, que serve como base para a formação de pessoal especializado em diversas áreas integrantes do grande campo de biodiversidade. No presente artigo, apresentamos um breve histórico do MZSP e um relato mais detalhado sobre a criação, a expansão e a qualificação do acervo da Coleção de Formigas do MZSP, que atualmente conta com quase 440 mil espécimes em via seca, além de amostras não quantificadas em via úmida.
\end{abstract}

Palavras-chave: Biodiversidade. Coleção científica. Entomologia. Mirmecologia. Prática curatorial.

\begin{abstract}
The Formicidae Collection of the Museu de Zoologia da Universidade de São Paulo (MZSP) is an invaluable representation of the ant's biodiversity, especially from the Neotropical Region. The collection was formed through the contribution of naturalists, authorities in myrmecology, by the results of scientific projects and by virtue of its role as a judicial keeper of ant samples gathered from different scientific studies. In addition to housing a significant number of type material, being 4,741 type specimens of 892 species widely distributed. The constant and adequate curatorial practices necessary for its maintenance and study is one of the major challenges faced by curators. These practices are also a basis for the training of specialized personnel, students and researchers in the biodiversity field. In this work, we present a brief history of the MZSP and a more detailed account of the formation, expansion and qualification of the Formicidae Collection of the MZSP, which currently houses almost 440 thousand pinned specimens, in addition to non-quantified samples in alcohol vials.
\end{abstract}

Keywords: Biodiversity. Curatorial practice. Entomology. Myrmecology. Scientific collection.

ULYSSÉA, M. A. \& C. R. F. BRANDÃO, 2020. Um acervo centenário: a história da Coleção de Formigas (Insecta: Hymenoptera: Formicidae) do Museu de Zoologia da Universidade de São Paulo. Boletim do Museu Paraense Emílio Goeldi. Ciências Naturais 15(1): 265-276. DOI: http://doi.org/10.46357/bcnaturais.v15i1.291.

Autora para correspondência: Mônica Antunes Ulysséa. Av. Nazaré, 481 - Ipiranga. São Paulo, SP, Brasil. CEP $04263-000$ (monicaulyssea@ gmail.com).

Recebido em 12/03/2020

Aprovado em 12/04/2020

Responsabilidade editorial: Lívia Pires do Prado
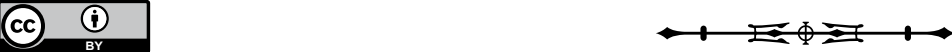


\section{BREVE HISTÓRICO SOBRE A FORMAÇÃO DO MZSP}

Museu de Zoologia da Universidade de São Paulo (MZSP) foi criado em 1969, mas a história do seu acervo é mais antiga do que a da própria universidade (fundada em 1934). Sua origem data de 1866, ano de criação da Comissão Geográfica e Geológica da Província de São Paulo (CGG), cujo objetivo era percorrer o território paulista visando reconhecer e avaliar os potenciais da região e expandir a cultura do café. $\bigcirc$ geólogo norte-americano Orville Adelbert Derby (1851-1915), então pesquisador do Museu Imperial e Nacional (Rio de Janeiro), foi nomeado chefe da comissão. Orville idealizou a criação e a organização de um museu com o acervo resultante das expedições da CGG, abrangendo as áreas de Geografia, História e Geologia (Vanzolini, 1994; Brandão \& Samara, 2005; Brandão \& Costa, 2007; Landim, 2011, 2018; MZSP, 2019; Nearns et al., 2019; Museu Paulista, 2020). Os dados sobre o material coligido pela CGG podem ser encontrados nos arquivos do Museu Geológico de São Paulo (MUGEO).

Paralelamente, o Museu Sertório, de propriedade do coronel Joaquim Sertório, foi organizado pelo naturalista sueco Johan Albert Constantin Löfgren (1854-1918) e possuía reconhecido valor mineralógico, zoológico, arqueológico, etnográfico e histórico. Seu acervo foi adquirido pelo conselheiro Francisco de Paula Mayrink (1839-1906), em 1890, e doado junto à Coleção Pessanha ao governo paulista, sendo adicionado ao acervo do Museu Provincial, pertencente à Sociedade Auxiliadora do Progresso da Província de São Paulo; a junção destes acervos formou uma grande coleção que ficou sob responsabilidade da CGG. Em 1891, foi criado o Museu do Estado, cuja direção interina ficou a cargo de Alberto Löefgren. Em janeiro de 1983, o museu passou a fazer parte da CGG, cujo presidente era Orville, e em agosto do mesmo ano o museu foi definido como um órgão independente da CGG, passando a denominar-se Museu Paulista (MP). Assim, todo o acervo oriundo da CGG, do Museu Sertório, da Coleção Pessanha e do Museu Provincial passou a formar o MP e a ocupar o palácio Monumento do Ipiranga (Vanzolini, 1994; Brandão \& Samara, 2005; Brandão \& Costa, 2007; Landim, 2011, 2018; MZSP, 2019; Nearns et al., 2019; Museu Paulista, 2020).

O primeiro diretor do MP (entre 1894 e 1915), indicado por Orville, foi Hermann Friedrich Albrecht von Ihering (1850-1930), zoólogo alemão, radicado no Brasil desde 1880, que havia atuado como naturalista-viajante do Museu Imperial e Nacional e como pesquisador da CGG. Em 1895, o MP abriu as portas para o público. No seu discurso de inauguração, Hermann von Ihering destacou o caráter científico da instituição. Na sua concepção, o museu deveria se concentrar nos objetivos de instruir e de contribuir para o progresso da ciência. A proposta de aprofundar a especialização do acervo foi colocada em prática a partir da coleta em várias regiões do país (o museu empregava reconhecidos naturalistas-viajantes, como Ernesto Garbe [1853-1925], constantemente em campo), de estudos comparativos e do intercâmbio com cientistas de diversas instituições e países. Hermann von Ihering priorizou os estudos zoológicos devido à sua formação. Durante sua gestão, em 1905, o acervo artístico do MP foi transferido e veio a constituir o acervo inicial da atual Pinacoteca do estado (Vanzolini, 1994; Brandão \& Samara, 2005; Brandão \& Costa, 2007; Landim, 2011, 2018; MZSP, 2019; Nearns et al., 2019; Museu Paulista, 2020).

Em 1916, foi nomeado como diretor do MP o advogado Armando Prado (1880-1956) e, posteriormente (1917), o historiador Afonso d'Escragnolle Taunay (18761958) assumiu o cargo. Neste momento, o MP assumiu sua nova vocação: preservar a História do Brasil. Na década de 1920, o MP foi reestruturado nas seções de História Nacional de São Paulo, de Zoologia e de Botânica. Em 1927, a Seção de Botânica foi transferida para o Instituto de Botânica, hoje ligado à Secretaria de Estado do Meio Ambiente (SEMA). Em 1939, a Seção de Zoologia tornou-se - Departamento de Zoologia da Secretaria de Agricultura, Indústria e Comércio do Estado de São Paulo (DZSP) e, em 1941, seu acervo passou a ser abrigado no prédio localizado no Parque da Independência, entre a avenida

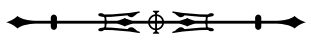


Nazaré e a rua Padre Marchetti; a partir desta época, passou a ser conhecido popularmente como "Museu dos Bichos". Em 1969, o Departamento de Zoologia foi incorporado à Universidade de São Paulo (USP) como Museu de Zoologia, sediado até hoje no mesmo prédio (Vanzolini, 1994; Brandão \& Samara, 2005; Brandão \& Costa, 2007; Landim, 2011, 2018; MZSP, 2019; Nearns et al., 2019; Museu Paulista, 2020). Recentemente, foi adicionada, temporariamente, uma parte do conjunto da Congregação das Irmãzinhas da Imaculada Conceição, na mesma avenida Nazaré, em frente ao Museu de Zoologia, onde são mantidas algumas coleções, laboratórios e parte da administração. $\bigcirc$ museu apresenta, atualmente, expressivas coleções nas áreas de Carcinologia, Chelicerata, Entomologia, Ictiologia, Invertebrados Marinhos, Herpetologia, Malacologia, Mastozoologia e Ornitologia.

O MZSP destaca-se, em escala mundial, pelo volume de suas coleções, que cobrem todo o reino animal, e pela qualidade da curadoria realizada ao longo de sua história de 154 anos (tendo como ponto de partida a criação da CGG), além das atividades de pesquisa, divulgação e formação de recursos humanos especializados em grau de excelência. O acervo científico do MZSP representa uma amostra inestimável da biodiversidade, principalmente da região neotropical. $O$ acervo do MZSP é produto da aquisição de importantes coleções de especialistas, permutas com outras instituições, doações e coletas realizadas através de expedições organizadas pela própria instituição ou por outras instituições que utilizam este museu como repositório de amostras (Vanzolini, 1994; Landim, 2018; MZSP, 2019). Nos anos mais recentes, o incremento do acervo tem ocorrido principalmente por doações, coletas organizadas no âmbito dos projetos acadêmicos realizados na instituição e através do depósito de material proveniente de estudos de impacto e monitoramento ambiental.

\section{COLEÇÃO DE FORMICIDAE DO MZSP}

A Coleção de Formicidae do MZSP, sediada no Laboratório de Hymenoptera da instituição, é uma das coleções mais importantes para a região neotropical, em razão do tamanho do acervo e por sua representatividade tanto de espécies quanto por cobrir uma área geográfica consideravelmente ampla (Brandão, 2000). São milhares de exemplares (cerca de 440.000, além de amostras em via úmida não quantificadas), oriundos de diversas localidades e representantes das 17 subfamílias atuais de Formicidae, sendo 4.741 espécimes-tipo de 892 espécies (dados dos tipos até 2017).

\section{A FORMAÇÃO INICIAL DO ACERVO DE FORMIGAS}

A Coleção de Formigas hoje abrigada no MZSP começou a ser formada ainda no MP. Os primeiros exemplares foram coletados no início do século XX pelo próprio diretor do museu, Hermann von Ihering, e pelos especialistas da instituição, destacando-se Hermann Luederwaldt (18651934) e Curt Schrottky (1874-1937). Sob orientação de Hermann von Ihering, o museu reuniu exemplares representativos das diversas ordens de insetos através do intercâmbio de material com museus europeus. Essa troca intensa permitiu a identificação por comparação da fauna brasileira e a descrição de muitas espécies de formigas por especialistas, como Gustav Mayr (1830-1908), Carlo Emery (1848-1925), Auguste Forel (1848-1931), William M. Wheeler (1865-1937), Felix Santschi (1872-1940), Carlo Menozzi (1848-1931) e Marion R. Smith (1894-1981). Várias espécies coletadas no Brasil foram descritas pelos autores ora citados, que, muitas vezes, enviavam os espécimestipo de volta a São Paulo, contribuindo para a formação e a qualificação da coleção.

Auguste Forel (1908) é autor da primeira publicação com extensivo material de formigas oriundo do MP, principalmente do estado de São Paulo, intitulada "Ameisen aus Sao Paulo (Brasilien), Paraguay etc". Neste artigo, Forel presta homenagem a Hermann von Iheringi, descrevendo seis espécies em seu nome: Camponotus iheringi, Crematogaster iheringi, Eciton iheringi (hoje Neivamyrmex), Ponera iheringi (atualmente Hypoponera), Solenopsis 
iheringi e Wasmannia iheringi. Nos anos seguintes, Forel homenageou-o outras quatro vezes: Leptogenys iheringi Forel, 1911, Megalomyrmex iheringi Forel, 1911, Dorymyrmex jheringi Forel, 1912 e Azteca iheringi Forel, 1915. Entretanto, cabe ressaltar que as primeiras homenagens ao zoólogo e exímio coletor foram feitas por Carlo Emery, a partir da descrição de espécies coletadas no Rio Grande do Sul: Cryptocerus jheringi (hoje Cephalotes) (Emery, 1894), Atta iheringi (hoje Mycetomoellerius) (Emery, 1888) e Rhopalothrix iheringi (hoje Octostruma) (Emery, 1888).

\section{CURADORES DA COLEÇÃO DE FORMIGAS}

O polonês Karol Lenko (1914-1975) trabalhou como curador da Coleção de Formigas entre 1960, quando o acervo fazia parte do DZSP, a 1969 (Nomura, 1991). Este período ficou marcado pela extensa permuta de material com o Museu Paraense Emílio Goeldi (MPEG) (convênio DZSP-MPEG, firmado na década de 1960) e com os freis franciscanos Thomas Borgmeier (1892-1975) e Walter Wolfgang Kempf (1920-1976), reconhecidos pela grande contribuição à Mirmecologia mundial, sobretudo na área da taxonomia.

Frei Borgmeier (1923, 1927) publicou catálogos das formigas do Brasil, atualizando uma das primeiras listas de espécies de formigas para o país, publicada por Forel (1895) a partir do material amazônico enviado pelo zoólogo suíço Emílio Augusto Goeldi (1859-1917), diretor do MPEG. Além disso, descreveu inúmeras espécies e formas ergatoides e dichthadiiformes; revisou gêneros como Atta (Fabricius, 1804), Eciton (Latreille, 1804), Labidus Jurine, 1807, Neivamyrmex (Borgmeier, 1940) e Nomamyrmex (Borgmeier, 1936); e, em 1931, fundou a Revista de Entomologia, cujo último volume, 22, foi publicado em 1951 (Klingenberg \& Brandão, 2005). Frei Kempf, por sua vez, realizou estudos taxonômicos e revisão total ou parcial de diversos gêneros como Anochetus (Mayr, 1861), Cephalotes (Latreille, 1802), Cyphomyrmex Mayr, 1862, Dinoponera (Roger, 1861), Heteroponera Mayr, 1877, Hylomyrma (Forel, 1912), Irogeria (Emery, 1915) (hoje Rogeria Emery, 1894), Leptothorax (Mayr, 1855), Megalomyrmex Forel, 1885,
Monacis (Roger, 1862) (hoje Dolichoderus [Lund, 1831]), Mycetarotes (Emery, 1913), Mycocepurus (Forel, 1893), Pachycondyla (Smith, 1858), Pheidole (Westwood, 1839), Procryptocerus (Emery, 1887), Pseudomyrmex (Lund, 1831), Rogeria, Strumigenys (Smith, 1860), Thaumatomyrmex Mayr, 1887 e Zacryptocerus (Wheeler, 1911) (hoje Cephalotes), descrevendo muitas espécies. Na década de 1960, publicou estudos em colaboração com William Louis Brown Jr. (19221997) sobre a tribo Basicerotini, os gêneros Acanthognathus Mayr, 1887 e Europhalothrix Brown \& Kempf, 1961, além de descrever junto com ele o gênero Tatuidris Brown \& Kempf, 1968. Borgmeier e Kempf ainda fundaram a revista científica Studia Entomologica, editada por Kempf até o volume 19 (1976). O último volume da revista, 20, foi editado por Lauro Pereira Travassos Filho (1918-1989) em 1978.

Com a aposentadoria de Karol Lenko, em 1969, a coleção de formigas passou por um período relativamente curto de dormência, até o início dos trabalhos de Carlos Roberto Ferreira Brandão junto à coleção, ainda em 1975, como estagiário e inicialmente mais interessado em estudos comportamentais.

No final da década de 1940, Borgmeier havia doado para o Frei Kempf sua coleção pessoal, que incluía espécimes, cadernos de campo e catálogos, sua biblioteca e seus equipamentos. No ano seguinte à morte inesperada de Kempf, em 1977, enquanto participava de um Congresso Internacional de Entomologia, na cidade de Washington, em que apresentaria suas conclusões sobre a distribuição geográfica das formigas do estado de São Paulo, a Coleção Borgmeier-Kempf foi comprada da Ordem Franciscana pelo MZSP, em nome do Dr. Nelson Papavero, chefe do serviço de entomologia do museu, com apoio do Conselho Nacional de Desenvolvimento Científico e Tecnológico (CNPq). O transporte da coleção da Universidade de Brasília (UNB), onde Frei Kempf atuou como professor visitante entre os anos de 1973 e 1976, para o MZSP foi financiado pela Fundação de Amparo à Ciência do Estado de São Paulo (FAPESP) (Klingenberg \& Brandão, 2005). Este importante acervo ampliou 
consideravelmente a então modesta Coleção de Formigas do museu, que ficava acondicionada em dois armários, sem maior organização interna.

Em 1981, o agora Dr. Carlos Roberto Ferreira Brandão, que fora estagiário do MZSP desde 1975, foi contratado como curador da Coleção de Hymenoptera do MZSP, ficando responsável pelo Laboratório de Hymenoptera. Brandão estabeleceu três linhas de pesquisa relacionadas: taxonomia e sistemática, ecologia de comunidades e evolução do comportamento social, dedicando-se, em especial, ao estudo de formigas nestas linhas. Entre suas contribuições para taxonomia, destacamse: a atualização do catálogo abreviado das formigas da região neotropical; a revisão dos gêneros Basiceros Schulz, 1906, Blepharidatta Wheeler, 1915, Kalathomyrmex Klingenberg \& Brandão, 2009, Lachnomyrmex Wheeler, 1910, Megalomyrmex, Mycetagroicus Brandão \& MayhéNunes, 2001, Mycetarotes, Mycetophylax (Emery, 1913), Oxyepoecus Santschi, 1926, Paramycetophylax (Kusnezov, 1956), Sphinctomyrmex Mayr, 1866, Stegomyrmex Emery, 1912 e Trachymyrmex (Forel, 1893) e da subfamília Leptanilloidinae; a descrição dos gêneros Asphinctanilloides Brandão et al., 1999, Cariridris Brandão \& Martins-Neto, 1990, Cyatta Sosa-Calvo et al., 2013, Mycetagroicus e Tropidomyrmex Silva et al., 2009; a descrição de espécies dos gêneros Adelomyrmex Emery, 1897, Belonopelta Mayr, 1870, Cariridris, Cephalotes, Cyatta, Gnamptogenys (Roger, 1863), Lachnomyrmex, Leptomyrmex (Mayr, 1862), Leptothorax, Megalomyrmex, Mycetagroicus, Oxyepoecus, Technomyrmex Mayr, 1872 e Tropidomyrmex; e os catálogos de tipos depositados na coleção de formigas do MZSP isoladamente ou em colaboração com diversos pesquisadores.

Como estratégia de curadoria, Brandão optou por concentrar seus esforços de coleta nos ecossistemas do leste do Brasil - Cerrado (início dos anos 80 até os 90), Mata Atlântica (anos 90) e Caatinga (início dos anos 90 e 2010-2012) -, sempre alternando projetos de ampliação da coleção com projetos de reorganização e incorporação dos acervos coletados na etapa anterior. Nesses projetos, foram incluídos estudantes e pesquisadores em vários níveis, de formação profissional, contribuindo, assim, com a realização de pesquisa nos mais variados âmbitos, como iniciação científica, trabalhos de conclusão de curso, dissertações, teses e pós-doutorados. As pesquisas foram fomentadas, em especial, pela FAPESP e pelo $\mathrm{CNPq}$, além de outras agências no Brasil e no exterior. Diferentes técnicas de coleta (busca ativa, extrator de Winkler, isca atrativa, pitfall) foram empregadas nesses distintos ambientes, mas que, pelo seu caráter quantitativo, permitiram, ao fim, comparação entre os sistemas.

\section{CERRADOS BRASILEIROS}

O projeto "Insetos sociais das formações vegetais do Nordeste brasileiro" (FAPESP, processo n. 1990/27756), focado nos biomas Caatinga e Cerrado, foi realizado em colaboração com a Dra. Eliana Marques Cancello, especialista em cupins (Isoptera) do MZSP, e o Dr. Paulo Oliveira, do Instituto de Biologia da Universidade de Campinas (UNICAMP). Além de distintas fitofisionomias da Caatinga e do Cerrado, foram amostradas áreas de Campo Rupestre (Tabela 1), utilizando iscas atrativas com sardinha em óleo no solo e na vegetação (2 m), considerando-se os períodos diurno e noturno. Ao todo, foram coletadas cerca de 700 espécies de formigas, e estimativas realizadas na época indicaram que o número real estaria próximo de 800 espécies. A oferta de iscas nos dois extratos e nos dos períodos atrai faunas significativamente distintas, evidenciando que as faunas são especializadas em extratos e ativas de acordo com o período do dia. Ainda, não foram detectadas diferenças significativas na composição de espécies de formigas entre as formações de Cerrado avaliadas e de Campo Rupestre (Brandão, 1995).

Alguns importantes estudos foram realizados com o material coletado. Entre eles, destacam-se as dissertações de Adriana Araújo Reis-Menezes, "Levantamento da fauna de formigas de uma localidade de Cerrado e dinâmica de visitação às iscas" (Reis-Menezes, 1998); Rogério Silvestre, "Levantamento da fauna de formigas de uma mancha 
de Cerrado no Estado de São Paulo" (Silvestre, 1995, 2000; Silvestre et al., 2003); e de Rogério Rosa da Silva, "Riqueza de formigas nos Cerrados e similaridade entre uma localidade no Planalto Central e duas no Sudeste do Brasil" (Silva, R., 1999; Silva, R., et al., 2004).

\section{MATA ATLÂNTICA}

O projeto temático "Riqueza e diversidade de Hymenoptera e Isoptera ao longo de um gradiente latitudinal na Mata Atlântica - a floresta pluvial do leste do Brasil", coordenado pelo Dr. Brandão, pela Dra. Eliana Cancello, ambos do MZSP, e pela Dra. Angélica Penteado-Dias, da Universidade Federal de São Carlos (UFSCAR), foi financiado pelo Programa Biota-FAPESP (processo 1998/0583-0). O objetivo principal deste projeto abrangente era estudar como as distintas biologias de 15 famílias selecionadas de
Hymenoptera, e também de Isoptera, se comportavam ao longo de um gradiente latitudinal, privilegiando-se do fato de que a Mata Atlântica ocorre praticamente ao longo de um eixo norte-sul no litoral leste do Brasil. Dadas as características desta floresta, 26 localidades protegidas (Tabela 2), representando duas altitudes em relação ao nível do mar (entre 0-100 m e 800-900 m), foram amostradas seguindo o mesmo delineamento amostral: distância das bordas em pelo menos 500 m, investigação da fauna da serapilheira utilizando-se o extrator de Winkler, além de coletas com armadilhas Malaise, pratos amarelos e varrição da vegetação.

Uma imensa quantidade de material foi coletada no decorrer de quase dez anos de projeto, e os resultados até hoje repercutem, pois tanto as formigas coletadas quanto espécies de outras ordens de insetos estão disponíveis no

Tabela 1. Localidades e tipo vegetacional amostrados durante o projeto "Insetos sociais das formações vegetais do Nordeste brasileiro" (anos 1990).

\begin{tabular}{|c|c|c|c|}
\hline Estado & Município & Localidade mais específica & Vegetação \\
\hline \multirow{5}{*}{ Bahia } & Andaraí & & $\begin{array}{l}\text { Mata densa, que chega a } 30 \text { m de altura, } \\
\text { com pouco estrato herbáceo e arbustivo, e } \\
\text { serapilheira densa em algumas partes }\end{array}$ \\
\hline & Itaberaba & & Caatinga arbórea e arbustiva \\
\hline & Maracás & & $\begin{array}{l}\text { Mata de Cipó (Caatinga arbórea) e "Geraes" } \\
\text { (mata dominada por Jurema sp.) }\end{array}$ \\
\hline & Mucugê & & Campo rupestre \\
\hline & Santa Rita de Cássia & & Caatinga arbórea \\
\hline \multirow{2}{*}{$\begin{array}{l}\text { Distrito } \\
\text { Federal }\end{array}$} & & $\begin{array}{l}\text { Reserva Ecológica do Instituto Brasileiro de } \\
\text { Geografia e Estatística (IBGE) }\end{array}$ & Cerrado \\
\hline & & Estação Ecológica Águas Emendadas & Cerrado sensu stricto \\
\hline \multirow{2}{*}{ Goiás } & Alvorada do Norte & & Cerrado aberto/campo sujo \\
\hline & Alto Paraíso de Goiás & & Campo rupestre \\
\hline \multirow{4}{*}{ Piauí } & Canto do Buriti PI & & Caatinga de lajedo \\
\hline & 10 km N Corrente & Fazenda Maracujá & Ecótono Caatinga-Cerrado \\
\hline & 20 km S Floriano & Buriti do Sol & Ecótono Caatinga-Cerrado \\
\hline & 5 km L Oeiras PI & Fazenda Talhada & Caatinga arbórea \\
\hline São Paulo & Cajurú & $\begin{array}{l}\text { Fazenda Santa Carlota }\left(21^{\circ} 18^{\prime}-21^{\circ} 27^{\prime} \mathrm{S} ;\right. \\
\left.\qquad 47^{\circ} 12^{\prime}-47^{\circ} 20^{\prime} \mathrm{W}\right)\end{array}$ & Cerrado \\
\hline
\end{tabular}

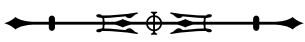


Tabela 2. Localidades da Mata Atlântica amostradas durante o projeto "Riqueza e diversidade de Hymenoptera e Isoptera ao longo de um gradiente latitudinal na Mata Atlântica - a floresta pluvial do leste do Brasil”, a partir de 1998 e nos anos 2000.

\begin{tabular}{|c|c|c|}
\hline Estado & Município & Localidade mais específica \\
\hline Alagoas & Quebrangulo & $09^{\circ} 19^{\prime} 18^{\prime \prime} \mathrm{S} / 36^{\circ} 28^{\prime} 32^{\prime \prime} \mathrm{W}$ \\
\hline \multirow{3}{*}{ Bahia } & Ilhéus & Mata da Boa Esperança (14 47’ 47" S/39॰ 03’ 56" W) \\
\hline & Mata São João & Reserva de Sapiranga ( $\left.12^{\circ} 58^{\prime} 16^{\prime \prime} \mathrm{S} / 38^{\circ} 30^{\prime} 39^{\prime \prime} \mathrm{W}\right)$ \\
\hline & Porto Seguro & E.E. Pau Brasil $\left(16^{\circ} 23^{\prime} 33^{\prime \prime} \mathrm{S} / 39^{\circ} 10^{\prime} 60^{\prime \prime} \mathrm{W}\right)$ \\
\hline \multirow{2}{*}{ Espírito Santo } & Santa Teresa & Estação Biológica Santa Lúcia (1958’ 09” S/40 32’ 15” W) \\
\hline & Sooretama & Reserva Biológica de Sooretama (19 04’ 21” S/3956’ 57” W) \\
\hline Paraíba & João Pessoa & Mata do Buraquinho $\left(07^{\circ} 08^{\prime} 24,7^{\prime \prime} \mathrm{S} / 34^{\circ} 51^{\prime} 33,2^{\prime \prime} \mathrm{W}\right)$ \\
\hline Pernambuco & Recife & Horto Dois Irmãos (08 $\left.00^{\prime} 32^{\prime \prime} \mathrm{S} / 34^{\circ} 56^{\prime} 40^{\prime \prime} \mathrm{W}\right)$ \\
\hline \multirow{2}{*}{ Paraná } & Morretes & Parque Estadual do Pau-Oco (25 34' 33,5” S/48 53' 19,5” W) \\
\hline & Tunas & Parque Estadual das Lauráceas (24 51' 16” S/48 43’00,4” W) \\
\hline \multirow{2}{*}{ Rio de Janeiro } & Nova Iguaçu & Reserva Biológica do Tinguá (22 34' 14" S/432 24' 51" W) \\
\hline & Santa Maria Madalena & 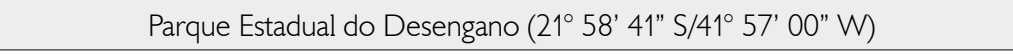 \\
\hline \multirow{4}{*}{ Santa Catarina } & Blumenau & Parque Estadual das Nascentes (270 06' 15" S/4909' 14” W) \\
\hline & Palhoça & Parque Estadual Serra do Tabuleiro (27 44' 28” S/48 41’ 50” W) \\
\hline & São Bento do Sul & Área de Proteção do Ambiental Rio Vermelho/Humbold (26 21' 51" S/49 16' 16" W) \\
\hline & São Bonifácio & Parque Estadual Serra do Tabuleiro (27 49’ 06” S/48 54' 41” W) \\
\hline \multirow{2}{*}{ Sergipe } & Areia Branca & Parque Estadual Serra de Itabaiana (10 45’ 54" S/37 19' 57,4” W) \\
\hline & Santa Luzia do Itanhy & Mata do Crasto $\left(11^{\circ} 22^{\prime} 39,3^{\prime \prime} \mathrm{S} / 37^{\circ} 25^{\prime} 07,4^{\prime \prime} \mathrm{W}\right)$ \\
\hline \multirow{8}{*}{ São Paulo } & Cananeia & Parque Estadual Ilha do Cardoso (250. $5^{\prime} 48,7^{\prime \prime} \mathrm{S} / 47^{\circ} 55^{\prime}$ 47,3” W) \\
\hline & Cunha & 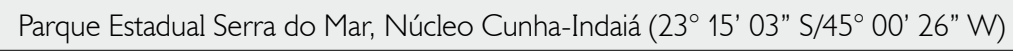 \\
\hline & Iguape & Estação Ecológica Jureia-Itatins, Núcleo Rio Verde (24 32’ 39” S/47 14’ 08” W) \\
\hline & Picinguaba & Parque Estadual Serra do Mar (23 $\left.20^{\prime} 10^{\prime \prime} \mathrm{S} / 44^{\circ} 50^{\prime} 15,3^{\prime \prime} \mathrm{W}\right)$ \\
\hline & Praia Grande & Parque Estadual Serra do Mar, Núcleo Pilões-Cubatão (23 58' 31" S/46 32' 24" W) \\
\hline & Ribeirão Grande & Parque Estadual Intervales, Base Barra Grande (24 18' 18” S/48 21' 55” W) \\
\hline & Salesópolis & Estação Biológica de Boraceia (23 31’ 56" S/45 50’ 47” W) \\
\hline & Tapiraí & 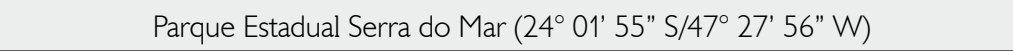 \\
\hline
\end{tabular}

acervo para estudo e foram utilizadas em vários trabalhos. Destacamos a tese de Rogério Rosa da Silva, "Estrutura de guildas de formigas de serapilheira (Hymenoptera: Formicidae) em quatro áreas de Floresta Atlântica do Sul e Sudeste do Brasil" (Silva, R., 2004; Silva, R. \& Brandão, 2010); a iniciação científica de Victor Alarcon, "Os Cerapachyinae (Formicidae) da Mata Atlântica" (Alarcon, 2013); e a dissertação de Thiago Sanches Ranzani da Silva, "Morfologia comparada das espécies da tribo Dacetini
(Hymenoptera: Formicidae) da Mata Atlântica" (Silva, T., 2014; Silva, T. \& Brandão, 2014). Ainda, o projeto "Limites geográficos e fatores causais de endemismo na Mata Atlântica em Diptera", também apoiado pelo Programa Biota-FAPESP, e coordenado pelo Dr. Dalton de S. Amorim (USP), não precisou coletar material e pôde obter todos os dados necessários para responder às questões a que se propunha estudar, utilizando material coletado pelo projeto de Hymenoptera-Isoptera.

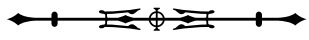




\section{CAATINGA}

A Caatinga, por muito tempo, representou um ecossistema cujo conhecimento era comparativamente muito incipiente, sendo aquele com a menor quantidade de informação para todos os grupos de invertebrados (Brandão, 1995; Brandão \& Yamamoto, 2004) e para formigas, em particular. As primeiras coletas se deram no início dos anos 1990, nos estados da Bahia e do Piauí, no âmbito do projeto "Riqueza e diversidade de Hymenoptera e Isoptera ao longo de um gradiente latitudinal na Mata Atlântica - a floresta pluvial do leste do Brasil" (Tabela 1).

No decorrer do tempo, Dr. Brandão estabeleceu parcerias com pesquisadores da Universidade Estadual do Ceará (UECE), Dr. Yves Quinet, e da Universidade Estadual de Feira de Santana (UEFS), Bahia, Dr. Gilberto de Nascimento. Com o último, foi firmada uma parceria de projeto através do Edital Casadinho (MCT/CNPq/ CT-Infra/CT-Petro/Ação Transversal IV n. 16/2008). Alguns dos resultados oriundos desta parceria foram a descrição da talvez única espécie de formiga, senão endêmica, pelo menos jamais coletada fora deste bioma, Oxyepoecus regularis Ulysséa \& Brandão, 2012, e a publicação da primeira listagem de formigas reconhecidas para este bioma - 173 espécies e uma subespécie, distribuídas em 11 subfamílias e 61 gêneros (Ulysséa \& Brandão, 2013a). Ambos os resultados são provenientes da dissertação de Mônica $A$. Ulysséa (Ulysséa, 2012; Ulysséa \& Brandão, 2012), cujas coletas foram realizadas no município de Milagres, Bahia (120 54,542' S/39० 51,279' W; 120 54,294' S/39० 52,083' W e $12^{\circ} 54,411^{\prime} S / 39^{\circ} 50,863^{\prime} \mathrm{W}$ ).

\section{EXPANSÃO E QUALIFICAÇÃO DO ACERVO DE FORMIGAS}

A coleção passou por três períodos principais de crescimento exponencial. Primeiro, a adição da Coleção Borgmeier-Kempf, seguida do acréscimo que ocorreu com o recebimento de material proveniente dos Cerrados-Mata Atlântica-Caatinga e, por último, e mais recentemente, o depósito de material oriundo de projetos de estudos de impacto ambiental e monitoramento de fauna, realizados em diversas regiões do país. Estes projetos são realizados por empresas de consultoria que, de acordo com as políticas estabelecidas pelos órgãos de controle ambiental (Sistema de Autorização e Informação em Biodiversidade/Instituto Chico Mendes de Conservação da Biodiversidade - SISBio/ICMBio), devem depositar o material biológico coletado em uma instituição brasileira responsável e devidamente capacitada para recebê-lo. Nesse sentido, a Coleção de Formigas recebeu um grande aporte de espécimes através dos projetos "Programa de monitoramento da Ferrovia Norte-Sul" (Goiás/Maranhão/ Tocantins), "Avaliações ecológicas rápidas do Centro-Leste do Tocantins", "Avaliações ecológicas rápidas do NordesteNoroeste do Tocantins" e "Programa de conservação da fauna silvestre na área de influência da Usina Hidrelétrica de Jirau - Entomofauna" (Rondônia). Além disso, a coleção cresce também com o depósito contínuo de material oriundo de projetos ligados a universidades e institutos de pesquisas, nacionais e internacionais.

Em 2015, Dr. Brandão foi contemplado com um auxílio no âmbito do Programa de Capacitação em Taxonomia (PROTAX), com financiamento tanto do CNPq (n. 440574/2015-3) quanto da FAPESP (processo n. 2016/50378-8) para desenvolvimento do projeto "Curadoria e qualificação da coleção de Hymenoptera do Museu de Zoologia da Universidade de São Paulo: formação de recursos humanos e ampliação do conhecimento sobre a biodiversidade brasileira". Destaca-se como principal objetivo deste projeto o uso da coleção como base para a formação de pessoal especializado principalmente nas áreas de taxonomia e sistemática. Cinco projetos de iniciação científica, um de mestrado e um de pós-doutorado com Hymenoptera foram realizados no âmbito do PROTAX, além de atividades curatoriais rotineiras, como avaliação da integridade e limpeza dos espécimes em via seca; revisão do acondicionamento, triagem, montagem e rotulagem de espécimes em via úmida; identificação dos espécimes no menor nível taxonômico possível; digitação 
das informações em planilha do banco de dados; visitas às coleções estrangeiras e nacionais para treinamento para identificação de espécimes; visita de pesquisadores à coleção do MZSP para o treinamento de pessoal e identificação de espécimes; e remessa de material a instituições para ser identificado por especialistas.

Um dos resultados de projetos desenvolvidos em taxonomia é a publicação de artigos com a designação de espécimes-tipo e o depósito dos mesmos em coleções de reconhecida importância para o grupo. A Coleção de Formigas do MZSP detém expressivos 4.741 espécimes-tipo de 892 espécies (dados até 2017). Estes dados são frutos da compilação dos oito catálogos de tipos publicados a partir de 2005 por Brandão e seus estudantes (Klingenberg \& Brandão, 2005; Scott-Santos et al., 2008; Brandão et al., 2010; Esteves et al., 2011; Ulysséa \& Brandão, 2013b; Prado \& Brandão, 2013; Ulysséa et al., 2015, 2017). Resta ainda atualizar esta lista a partir de descrições mais recentes, publicadas após os catálogos de tipos correspondentes.

Deve-se pontuar a contribuição de muitos colaboradores voluntários, técnicos com distintas formações e níveis de especialização, e inúmeros estudantes e estagiários, que cooperaram na construção, guarda e qualificação deste importante acervo, fonte para pesquisadores e estudantes de muitas procedências. Nos anos mais recentes, destacam-se as contribuições de Bodo Dietz, Christiana Kligenberg, Christiane Yamamoto, Cristiane Scott-Santos, Emília Albuquerque, Flávia Esteves, Lívia Prado, Mônica A. Ulysséa, Nicolas Albuquerque, Rodolfo Probst, Rodrigo Feitosa, Rogério R. da Silva, Rogério Silvestre e Thiago Sanches Ranzani da Silva, que desenvolveram estudos sob orientação do Dr. Brandão. Ainda, como a coleção é fonte importante de dados para pesquisadores e estudantes de muitas procedências, o laboratório envia exemplares por empréstimo e também recebe inúmeros visitantes. Desde 2015, recebemos a visita de 33 discentes, oito docentes e um pesquisador de instituições nacionais (Instituto de Biociências-USP, MPEG, Pontifícia Universidade Católica de Campinas -
PUC-Campinas, Universidade Federal do Pará - UFPA, Universidade Federal do Paraná - UFPR, Universidade Federal Rural do Rio de Janeiro - UFRRJ, Universidade Federal de Viçosa - UFV, Universidade Estadual Paulista Júlio de Mesquita Filho de Rio Claro - UNESP-Rio Claro, Universidade Federal do Vale do São Francisco - UNIVASF e Universidade Mogi das Cruzes - UMC) e internacionais (Centro Regional de Investigaciones Científicas y Transferencia Tecnológica de La Rioja/Argentina, Museu Nacional de História Natural e da Ciência de Lisboa/Portugal, Smithsonian National Museum of Natural History/USA - NMHNSmithsonian, The Ohio State University/USA, University of California, Davis/USA - UC-Davis, Universidad Nacional de Colombia/Colômbia - UNAL e The University of Utah/ USA), que passam períodos estudando o acervo como parte de suas pesquisas e, consequentemente, auxiliam na qualificação da coleção, aprimorando a identificação dos exemplares. Este aprimoramento também é feito a partir de empréstimos de material.

\section{O ACERVO NOS DIAS DE HOJE}

Desde setembro de 2018, a Coleção de Formiga do MZSP está sendo reorganizada à luz de novas práticas curatoriais e das mais recentes propostas de classificação de Formicidae com apoio da FAPESP, através do projeto temático "Coletar, identificar, processar, difundir: o ciclo curatorial e a produção do conhecimento" (processo n. 2017/07366-1). A pesquisadora de pós-doutorado Mônica A. Ulysséa vem se dedicando a esta reorganização junto com a estudante Gabriella Chimenti (bolsista de IC FAPESP) e a estagiária Larissa Jerez (bolsista técnica FAPESP).

A coleção está organizada em três acervos: seco, úmido e molecular. O acervo seco, que abrange sobretudo espécimes colados em triângulos de papel alfinetados, está organizado em armários compactadores de metal que abrigam 914 gavetas entomológicas. Os 389.418 (contagem manual por Gabriela Chimenti, Larissa Jerez e Mônica Ulysséa, terminada em fevereiro de 2020) espécimes em via seca (Tabela 3) estão distribuídos nas gavetas em caixas 
Tabela 3. Número de exemplares do acervo seco e organizado da Coleção de Formicidae do MZSP.

\begin{tabular}{|c|c|}
\hline Subfamília de Formicidae & Número de exemplares \\
\hline Agroecomyrmecinae & 7 \\
\hline Amblyoponinae & 1.175 \\
\hline Aneuretinae & 3 \\
\hline Apomyrminae & 2 \\
\hline Dolichoderinae & 24.552 \\
\hline Dorylinae & 21.131 \\
\hline Ectatomminae & 14.772 \\
\hline Formicinae & 76.546 \\
\hline Heteroponerinae & 1.460 \\
\hline Leptanillinae & 10 \\
\hline Martialinae & 1 \\
\hline Myrmeciinae & 45 \\
\hline Myrmicinae & 207.758 \\
\hline Paraponerinae & 882 \\
\hline Ponerinae & 27.149 \\
\hline Proceratinae & 762 \\
\hline Pseudomyrmecinae & 13.163 \\
\hline Total de exemplares & 389.418 \\
\hline
\end{tabular}

de papelão com fundo de isopor, dispostas em ordem alfabética por subfamília-gênero-espécie e por localidade, além de 101 gavetas com cerca de 30 mil espécimes, que estão em processamento para associação de exemplares, confecção de rótulos de localidade e/ou identificação, e identificação em subfamília-gênero-espécie. $\bigcirc$ acervo úmido, conservado em álcool 70\% a 96\%, conta com 804 potes com no máximo 20 amostras cada, acondicionadas em frascos de vidro transparente, com batoque e tampa de rosca, sendo majoritariamente réplicas do material montado, além de exemplares em estágios imaturos. Já o incipiente acervo molecular, iniciado em 2015, conta com 155 amostras guardadas em freezer.

\section{PERSPECTIVAS FUTURAS}

Nos últimos anos, os esforços estão concentrados na reorganização e qualificação do acervo. Uma vez formada uma coleção, a sua curadoria precisa ser constante para a melhor salvaguardada da biodiversidade ali representada. Este é um dos grandes desafios das coleções, que enfrentam dificuldades para manter seus acervos em condições adequadas, por falta de técnicos especializados, de infraestrutura e em razão da escassez de recursos financeiros, derivada tanto de períodos de corte de verba quanto de 'abundância' de financiamento (Zaher \& Young, 2003; Santos et al., 2018).

A Coleção de Formigas do MZSP tem grande valor histórico e científico e, como parte das importantes coleções biológicas do mundo, é fonte fundamental para o desenvolvimento de inúmeros estudos por deter os elementos básicos (táxons) para o entendimento do próprio táxon, dos biomas onde estão inseridos e das relações que estabelecem com outros organismos. Neste sentido, esperamos que a ciência e, por conseguinte, a educação sejam priorizadas no país, independente dos partidos políticos que o governam.

\section{AGRADECIMENTOS}

Agradecemos à Dione Seripierri, bibliotecária do MZSP, que auxiliou na averiguação das datas apresentadas, e aos três revisores anônimos, pela leitura crítica do artigo, pelas correções e sugestões. M. A. Ulysséa agradece à FAPESP (processos n. 2012/21309-7, 2015/06485-1, 2018/11453-0, 2019/24810-8). C. R. F. Brandão agradece ao PROTAX/ CNPq (440574/2015-3), à FAPESP (PROTAX/FAPESP processo n. 2016/50378-8, TEMÁTICO processo $n$. 2017/07366-1), ao Instituto Nacional de Ciência e Tecnologia dos Hymenoptera Parasitoides da Região Sudeste Brasileira (INCT-HYMPAR) (CNPq/FAPESP/CAPES).

\section{REFERÊNCIAS}

ALARCON, V. I. A., 2013. Os Cerapachyinae (Formicidae) da Mata Atlântica. Iniciação Científica (Graduação em Ciências Biológicas) - Instituto de Biociências, Universidade de São Paulo, São Paulo.

BORGMEIER, T., 1923. Catalogo systematico esynonymico dasformigas do Brasil. 1 parte. Subfam. Dorylinae, Cerapachyinae, Ponerinae, Dolichoderinae. Archivos do Museu Nacacional 24: 33-103.

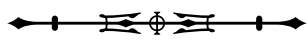


BORGMEIER, T., 1927. Catalogo systematico e synonymico das formigas do Brasil. 2 parte. Subf. Pseudomyrminae, Myrmicinae, Formicidae. Archivos do Museu Nacacional 29: 69-164.

BRANDÃO, C. R. F., 1995. Formigas dos Cerrados e Caatingas. Tese de Livre-docência - Universidade de São Paulo, São Paulo.

BRANDÃO, C. R. F., 2000. Major regional and type collections of ants (Formicidae) of the world and sources for the identification of ant species. In: D. AGOSTI, J. D. MAJER, L. E. ALONSO \& T. R. SCHULTZ (Ed.): Ants: standard methods for measuring and monitoring biodiversity: 172-185 Smithsonian Institution Press, Washington. DOI: http://doi.org/10.5281/zenodo.11736.

BRANDÃO, C. R. F. \& C. I. YAMAMOTO, 2004. Invertebrados da Caatinga. In: J. M. CARDOSO, M. TABARELLI, M. T. FONSECA \& L. V. LINS (Org.): Biodiversidade da Caatinga: áreas e ações prioritárias para a conservação: 135-140. MMA/EFPE/Conservação Internacional do Brasil/EMBRAPA, Brasília.

BRANDÃO, C. R. F. \& E. M. SAMARA, 2005. Da difusão à pesquisa no Estado de São Paulo. A história do Museu Paulista da Universidade de São Paulo. Revista de Cultura e Extensão da USP (0): 94-103.

BRANDÃO, C. R. F. \& C. COSTA, 2007. Uma crônica da integração dos museus estatutários à USP. Anais do Museu Paulista 15(1): 207-217. DOI: http://doi.org/10.1590/S0101-47142007000100005.

BRANDÃO, C. R. F., F. A. ESTEVES \& L. P. PRADO, 2010. A catalogue of the Pseudomyrmecinae ants type specimens (Hymenoptera, Formicidae) deposited in the Museu de Zoologia da Universidade de São Paulo, Brazil. Papéis Avulsos de Zoologia 50(45): 693-699. DOI: http://doi.org/10.1590/S0031-10492010004500001.

ESTEVES, F. A., C. R. F. BRANDÃO \& L. P. PRADO, 2011. The type specimens of "dorylomorph" ants (Hymenoptera, Formicidae: Aenictinae, Ecitoninae, Cerapachyinae, Leptanilloidinae) deposited in the Museu de Zoologia da Universidade de São Paulo, Brazil. Papéis Avulsos de Zoologia 51(22): 341-357. DOI: http://doi.org/10.1590/ S0031-10492011002200001.

FOREL, A., 1895. A fauna das formigas do Brazil. Boletim do Museu Paraense de Historia Natural e Ethnographia 1: 89-139.

FOREL, A., 1908. Ameisen aus Sao Paulo (Brasilien), Paraguay etc. gesammelt von Prof. Herm. v. Ihering, Dr. Lutz, Dr. Fiebrig, etc. Verhandlungen der Kaiserlich-Königlichen ZoologischBotanischen Gesellschaft in Wien 58: 340-418.

KLINGENBERG, C. \& C. R. F. BRANDÃO, 2005. The type specimens of fungus growing ants, Attini (Hymenoptera, Formicidae, Myrmicinae) deposited in the Museu de Zoologia da Universidade de São Paulo, Brazil. Papéis Avulsos de Zoologia 45(4): 41-50. DOI: http://doi.org/10.1590/S0031-10492005000400001.

LANDIM, M. I., 2011. Museu de Zoologia da Universidade de São Paulo: adaptação aos novos tempos. Estudos Avançados 25(73): 205-216. DOI: http://doi.org/10.1590/S0103-40142011000300023.
LANDIM, M. I., 2018. Um oceano de desconhecimento sobre a biodiversidade. Revista Museologia \& Interdisciplinaridade 7(14): 88-106. DOI: https://doi.org/10.26512/museologia.v7i14.18388.

MUSEU PAULISTA, 2020. Dicionário histórico-biográfico das ciências da saúde no Brasil (1832-1930). Disponível em: http:// www.dichistoriasaude.coc.fiocruz.br/iah/pt/verbetes/muspaul.htm. Acesso em: 27 fevereiro 2020

MUSEU DE ZOOLOGIA DA UNIVERSIDADE DE SÃO PAULO (MZSP), 2019. MZSP - Museu de Zoologia da Universidade de São Paulo. [Material institucional]. São Paulo.

NEARNS, E. H., F. E. L. NASCIMENTO \& S. A. CASARI, 2019. Onciderini Thomson, 1860 (Coleoptera: Cerambycidae: Lamiinae) types of the Museu de Zoologia, Universidade de São Paulo(MZSP), with a brief history of the Coleoptera collection. Insecta Mundi 715: 1-27.

NOMURA, H., 1991. Karol Lenko. In: H. NOMURA. Vultos da zoologia brasileira: v. 2: 204-205. Fundação Guimarães Duque (Coleção Mossoroense, Série C), Mossoró.

PRADO, L. P. \& C. R. F. BRANDÃO, 2013. Catalogue of Cephalotini ant types (Hymenoptera, Formicidae, Myrmicinae) deposited in the Museu de Zoologia da Universidade de São Paulo, Brazil. Papéis Avulsos de Zoologia 53(20): 187-209. DOI: http://doi.org/10.1590/ S0031-10492013002000001.

REIS-MENEZES, A. A., 1998. Levantamento da fauna de formigas de uma localidade de cerrado e dinâmica de visitação às iscas. Dissertação (Mestrado em Zoologia) - Instituto de Biociências, Universidade de São Paulo, São Paulo.

SANTOS, H. R. S., P.S. GÔLO, M. SILVA, I. S. COELHO, S. R. PAIVA\& D. R. OLIVEIRA, 2018. Os impactos da legislação ambiental brasileira sobre as coleções biológicas. Diversidade e Gestão 2(2): 52-61.

SCOTT-SANTOS, C. P., F. A. ESTEVES \& C. R. F. BRANDÃO, 2008. Catalogue of "poneromorph" ant type specimens (Hymenoptera, Formicidae) deposited in the Museu de Zoologia da Universidade de São Paulo, Brazil. Papéis Avulsos de Zoologia 48(11): 75-88. DOI: http://doi.org/10.1590/S0031-10492008001100001.

SILVA, R. R., 1999. Riqueza de formigas (Hymenoptera: Formicidae) nos cerrados e similaridade entre uma localidade no Planalto Central e duas no Sudeste do Brasil. Dissertação (Mestrado em Zoologia) - Instituto de Biociências, Universidade de São Paulo, São Paulo.

SILVA, R. R., 2004. Estrutura de guildas de formigas (Hymenoptera: Formicidae) de serapilheira em quatro áreas de Floresta Atlântica do Sul e Sudeste do Brasil. Tese (Doutorado em Zoologia) Instituto de Biociências, Universidade de São Paulo, São Paulo.

SILVA, R. R., C. R. F. BRANDÃO \& R. SILVESTRE, 2004. Similarity between Cerrado localities in Central and Southeastern Brazil based on the dry season bait visitors ant fauna. Studies on Neotropical Fauna and Environment 39(3): 191-199. DOI: https://doi.org/10.1 080/01650520412331271783

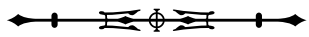


SILVA, R. R. \& C. R. F. BRANDÃO, 2010. Morphological patterns and community organization in leaf-litter ant assemblages. Ecological Monographs 80(1): 107-124. DOI: http://doi.org/10.1890/08-1298.1.

SILVA, T. S. R., 2014. Dacetini (Hymenoptera: Formicidae) da Mata Atlântica. Dissertação (Mestrado em Sistemática, Taxonomia Animal e Biodiversidade) - Museu de Zoologia, Universidade de São Paulo, São Paulo. DOI: http://doi.org/10.11606/D.38.2014. tde-06092014-121842.

SILVA, T. S. R. \& C. R. F. BRANDÃO, 2014. Further ergatoid gyne records in the ant tribe Dacetini (Formicidae: Myrmicinae). Neotropical Entomology 43(2): 161-71. DOI: http://doi. org/10.1007/s13744-013-0192-7.

SILVESTRE, R., 1995. Levantamento da fauna de formigas de uma mancha de Cerrado no estado de São Paulo e observações sobre a dinâmica de visitação às iscas. Dissertação (Mestrado em Entomologia) - Universidade de São Paulo, Ribeirão Preto, São Paulo.

SILVESTRE, R., 2000. As formigas dos Cerrados. Tese(Doutorado em Entomologia) - Universidade de São Paulo, Ribeirão Preto, São Paulo.

SILVESTRE, R., C. R. F. BRANDÃO \& R. R. SILVA, 2003. Grupos funcionales de hormigas: el caso de los gremios del cerrado. In: F. FERNÁNDEZ (Ed.): Introducción a las hormigas de la región Neotropical: 113-148. Instituto de Investigación de Recursos Biológicos Alexander Von Humboldt, Bogotá, Colombia.

ULYSSÉA, M. A., 2012. Estrutura de guildas em formigas associadas à serrapilheira de Caatingas arbóreas. Dissertação (Mestrado em Zoologia) - Universidade Estadual de Feira de Santana, Feira de Santana.

ULYSSÉA, M. A. \& C. R. F. BRANDÃO, 2012. A new ant species of Oxyepoecus (Hymenoptera: Formicidae: Myrmicinae), with the description of Oxyepoecus browni gyne and new records for the genus. Papéis Avulsos de Zoologia 52: 167-173. DOI: http://doi. org/10.1590/S0031-10492012001400001.
ULYSSÉA, M. A. \& C. R. F. BRANDÃO, 2013a. Ant species (Hymenoptera, Formicidae) from the seasonally dry forests of northeastern Brazil: a compilation from field surveys in Bahia and literature records. Revista Brasileira de Entomologia 57(2): 217 224. DOI: http://doi.org/10.1590/S0085-56262013005000002.

ULYSSÉA, M. A. \& C. R. F. BRANDÃO, 2013b. Catalogue of Dacetini and Solenopsidini ant type specimens (Hymenoptera, Formicidae, Myrmicinae) deposited in the Museu de Zoologia da Universidade de São Paulo, Brazil. Papéis Avulsos de Zoologia 53(14): 187-209. DOI: http://doi.org/10.1590/S003110492013001400001.

ULYSSÉA, M. A., L. P. PRADO \& C. R. F. BRANDÃO, 2015. Type specimens of the traditional Myrmicinae (Hymenoptera: Formicidae) ant tribes deposited in the Museu de Zoologia da Universidade de São Paulo, Brazil: Adelomyrmecini, Basicerotini, Blepharidattini, Crematogastrini, Formicoxenini, Lenomyrmecini, Myrmicini, Phalacromyrmecini, Pheidolini, Stegomyrmecini, Stenammini and Tetramoriini. Papéis Avulsos de Zoologia 55(12): 75-204. DOI: http://doi.org/10.1590/0031-1049.2015.55.12.

ULYSSÉA, M. A., L. P. PRADO \& C. R. F. BRANDÃO, 2017. Catalogue of the Dolichoderinae, Formicinae and Martialinae (Hymenoptera: Formicidae) types deposited at the Museu de Zoologia da Universidade de São Paulo, Brazil. Papéis Avulsos de Zoologia 57(23): 295-311. DOI: http://doi.org/10.11606/00311049.2017.57.23.

VANZOLINI, P. E., 1994. Museu de Zoologia. Estudos Avançados 8(22): 579-580. DOI: http://doi.org/10.1590/S010340141994000300085.

ZAHER, H. \& P. S. YOUNG, 2003. As coleções zoológicas brasileiras: panorama e desafios. Ciência e Cultura 55(3): 24-26. 